\title{
Accurate monitoring of terrestrial aerosols and total solar irradiance: the NASA Glory mission
}

\author{
Brian Cairns*a ${ }^{\text {a }}$, Greg Kopp ${ }^{\mathrm{b}}$, Michael Mishchenko ${ }^{\mathrm{a}}$, Hal Maring ${ }^{\mathrm{c}}$, Bryan Fafaul ${ }^{\mathrm{d}}$, Steven Pszcolka ${ }^{\mathrm{d}}$ \\ ${ }^{a}$ NASA Goddard Institute for Space Studies, 2880 Broadway, New York, NY, USA 10025; \\ ${ }^{\mathrm{b}}$ Laboratory for Atmospheric and Space Physics, 1234 Innovation Dr. \\ Boulder, CO, USA 80303; \\ ${ }^{\mathrm{c}}$ NASA Headquarters, 300 E Street SW, Washington, D.C., USA 20024-3210; \\ ${ }^{\mathrm{d}}$ NASA Goddard Space Flight Center, 8800 Greenbelt Road, Greenbelt, MD, USA 20771
}

\begin{abstract}
Current uncertainties in the total solar irradiance (TSI) and aerosol radiative forcings of climate are so large that they limit quantitative evaluation of climate models against global temperature change. Reducing these uncertainties is the objective of the NASA Glory mission scheduled for launch in November 2010 as part of the NASA A-Train. Glory is intended to meet the following scientific objectives: Improve the quantification of solar variability by continuing the uninterrupted 32-year satellite measurement record of TSI, facilitate the quantification of the aerosol direct and indirect forcings of climate, and provide better aerosol representations for use by other operational satellite instruments.
\end{abstract}

Keywords: Climate, total solar irradiance, aerosols, clouds, radiative forcing

\section{INTRODUCTION}

The Earth's climate depends upon the balance between incident solar radiation and the response of the atmosphere and surface via absorption, reflection, and re-radiation. Long-term changes in the composition of the atmosphere or the brightness of the Sun can cause global climate change and thereby affect local weather patterns and impact the quality of human life. The composition of the atmosphere is influenced by both natural and anthropogenic effects, such as volcanic eruptions and the byproducts of modern industrial societies. Over the past century the average temperature at the Earth's surface has increased by approximately $0.7^{\circ} \mathrm{C}$. Accurately attributing the portions of this increase and the concomitant climate change due to natural events or anthropogenic sources is of primary importance to the establishment of scientifically and economically effective policy.

Both natural and anthropogenic aerosols are important constituents of the atmosphere affecting global temperature. Although the climate effects of aerosols are believed to be nearly comparable to those of the green-house gases, they remain poorly quantified and represent the largest uncertainty regarding climate change. Numerous recent studies have indicated that the current uncertainties in the aerosol forcings are so large that they limit the requisite climate model evaluation by comparison with observed global temperature change. These uncertainties must be reduced significantly for uncertainty in climate sensitivity to be adequately constrained. Helping to address this overarching objective is the main purpose of the Aerosol Polarimetry Sensor (APS) on-board the NASA Glory mission.

The Glory's Total Irradiance Monitor (TIM) measures total solar irradiance (TSI), the Sun's spatially- and spectrallyintegrated radiative input that drives the Earth's climate. The Glory mission is intended to continue the TSI climate data record, which is now over 32 years long with continuity provided by several spacecraft missions, and to overlap with the similar TIM instrument currently operating on NASA's SOlar Radiation and Climate Experiment (SORCE) ${ }^{1}$.

The Sun's radiation provides nearly 10,000 times more energy to the Earth's climate system than any other source, so even small variations in this relatively stable input make TSI a climate forcing agent. In allocating the causes of climate change, sensitivities to different forcing agents must be determined, and these rely on long-term records of the input sources. Since TSI varies by approximately $0.1 \%$ over an 11 -year solar cycle and by unknown and potentially smaller 
amounts over multi-decadal time scales, the instruments acquiring these data must have exceptional long-term stability and accuracy.

The NASA Glory mission is a remote-sensing Earth-orbiting observatory that will carry the TIM and APS sensors and is scheduled for launch in November 2010 as part of the A-Train constellation of Earth-orbiting satellites ${ }^{2}$.

\section{SCIENTIFIC OBJECTIVES OF THE GLORY MISSION}

The Glory APS is intended to meet the following three scientific objectives:

- Facilitate the quantification of the aerosol direct and indirect effects on climate for use in climate models by determining the global distribution of the optical thickness and microphysical properties of natural and anthropogenic aerosols and clouds with much improved accuracy.

- Provide better aerosol representations for use in aerosol property retrieval by other operational satellite instruments.

- Provide an improved framework for the formulation of future comprehensive satellite missions for aerosol, cloud, and ocean color research.

The aerosol measurement requirements include the retrieval of the total column optical thickness (OT) and average column values of the effective radius and effective variance, the real part of the refractive index, and the singlescattering albedo. Since the aerosol population is typically bimodal, all these parameters must be determined for each mode. The refractive index must be determined at multiple wavelengths in a wide spectral range since this is the only means of constraining aerosol chemical composition from space. An integral part of the retrieval procedure must be the detection of non-spherical aerosols such as dust-like and soot particles since non-sphericity can significantly affect the results of optical thickness, refractive index, and size retrievals. The respective minimum cloud measurement requirements include the retrieval of the column cloud OT and the average column cloud droplet size distribution as well as the determination of the cloud phase and particle non-sphericity.

The Glory TIM scientific objectives are the continuity of the TSI record from SORCE with the required performance of:

- Accuracy 100 parts per million (ppm) (1 sigma)

- Stability $10 \mathrm{ppm} /$ year (1 sigma)

It is only by having an extended record of accurate TSI measurements that methods for extending the solar irradiance record into the past using proxies such as sunspot number and cosmogenic isotopes can be effective. Historic estimates of TSI are invaluable for determining climate sensitivities to solar forcing over thousands of years by comparisons with paleographic climate records.

\section{APS DESIGN}

The unique APS design takes full advantage of the extreme sensitivity of high-accuracy polarization data to aerosol and cloud particle microphysics. The key measurement requirements for the retrieval of aerosol and cloud properties from photopolarimetric data are high accuracy, a broad spectral range, and observations from multiple angles, including a method for reliable and stable calibration of the measurements. The APS built for the Glory mission by Raytheon $[3,4]$ meets these requirements.

The measurement approach required to ensure high accuracy in polarimetric observations employs a Wollaston prism. The field stop constrains the APS instantaneous field of view (IFOV) to $8 \pm 0.2 \mathrm{mrad}$, which, at the nominal Glory altitude of $705 \mathrm{~km}$, yields a geometric IFOV of $5.6 \mathrm{~km}$ at nadir. The spatial field is defined by the relay telescope and is collimated prior to the polarization separation provided by the Wollaston prism. This method guarantees that the measured orthogonal polarization states come from the same scene at the same time and allows the required polarimetric accuracy of $0.2 \%$ to be attained. To measure the Stokes parameters that define the state of linear polarization (I, $Q$, and $U$ ), the APS employs a pair of telescopes with one telescope measuring $I$ and $Q$ and the other telescope measuring $I$ and 
$U$. The broad spectral range of the APS is provided by dichroic beam splitters and interference filters that define nine spectral channels centered at the wavelengths $\lambda=410,443,555,670,865,910,1378,1610$ and $2250 \mathrm{~nm}$. Blue enhanced silicon detectors are used in the visible and near-IR channels, while $\mathrm{HgCdTe}$ detectors, passively cooled to $160 \mathrm{~K}$, are used in the short-wave IR channels and offer the very high signal-to-noise ratio required to yield a polarimetric precision better than $0.3 \%$ for typical clear sky scenes over the dark ocean surface.

All spectral channels except those at 910- and 1378-nm are free of strong gaseous absorption bands. The 1378-nm spectral channel is centered in a strong water vapor absorption band and is specifically intended for characterization of thin cirrus clouds and stratospheric aerosols. The 910-nm channel is also in a water vapor absorption band and will be used to provide information on the column water vapor amount. The locations of the other APS spectral channels are consistent with an optimized aerosol retrieval strategy because they take advantage of several natural circumstances such as the darkness of the ocean at longer wavelengths in the visible and near-infrared, the lower land albedo at shorter visible wavelengths, and the potential for using the 2200-nm band to characterize the land surface contribution at visible wavelengths.

The ability to view a scene from multiple angles is provided by scanning the APS IFOV along the spacecraft ground track with a rotation rate of 40.7 revolutions per minute with angular samples acquired every $8 \pm 0.4 \mathrm{mrad}$, thereby yielding $\sim 250$ scattering angles over the scene viewing angular range of $+50 /-62^{\circ}$. The polarization-compensated scanner assembly includes a pair of matched mirrors operating in an orthogonal configuration and has been demonstrated to yield instrumental polarization less than $0.05 \%$. The APS viewing angle range at the Earth is from $+60^{\circ}$ with respect to nadir to the limb in that aft direction. The scanner assembly also allows a set of calibrators to be viewed over that part of the scan range that does not view the Earth. These reference assemblies provide calibration of the zero of the polarimetric scale (unpolarized reference assembly, URA) and the scale factor for the polarimetric scale (polarized reference assembly, PRA). The zero of the radiometric scale is provided by the dark reference assembly (DRA) and the initial radiometric scale is defined by viewing a Spectralon ${ }^{\mathrm{TM}}$ plaque with a well characterized BRDF (solar reference assembly, SRA) and is subsequently tracked monthly using observations of the moon to ensure that aerosol and cloud retrieval products are stable over the period of the mission. Since the capabilities of the reference assemblies are crucial to meeting the measurement accuracy requirements for the APS their performance is presented in greater detail below.

\subsection{Unpolarized reference assembly}

The Unpolarized Reference Assembly (URA) provides intra-telescope relative responsivity factors, which are critical to meet polarization accuracy and stability. The URA is nadir viewing so that the spectral content and dynamic range of its depolarized scene look is virtually the same as that observed in the actual scene viewing observations. It provides a depolarized view for all of the detectors by means of a polarization scrambler composed of two wedges of crystalline quartz with the optical axes orientated $45^{\circ}$ to each other - a spatially varying retardance is created which scrambles any scene polarization. An additional flat fused silica element is used to reduce the effects of reflections.

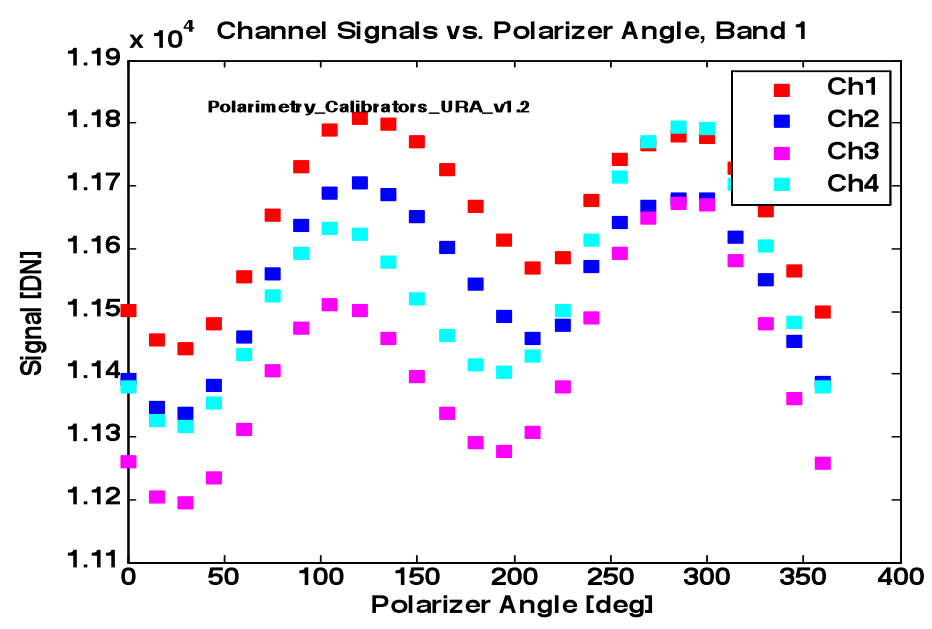

Figure 1: Example of the URA signal with a polarized source 
The URA accuracy was measured following the radiometric and calibration tests of the instrument. URA signal data was obtained with a completely polarized and unpolarized sources. The unpolarized signals provided data to calculate the relative gain coefficients $\mathrm{K} 1$ and $\mathrm{K} 2$. The polarized signal was generated using an ultra-high contrast wire grid polarizer inserted into the optical path between the APS and an integrating sphere and rotated $0^{\circ}-360^{\circ}$ in $15^{\circ}$ steps. The wire grid polarizer provides a full field polarized signal with uniform polarization azimuth and was found to provide extinction efficiencies of better than 1 in 1000 at $410 \mathrm{~nm}$ which is the spectral domain where the performance is worst.

The URA signals showed low modulation (less then $0.8 \%$ for $100 \%$ input being the worst case at $670 \mathrm{~nm}$ ), as shown in the example of Figure 1. By screening the scenes used for polarimetric calibration to bright clouds and snow fields that have a polarization of less than $10 \%$, even for the rainbow scattering angles of water clouds, the worst case polarization viewed through the URA can be reduced to less than $0.08 \%$ with a median value of $0.03 \%$.

The trend of the URA DoLP was evaluated over the sensor performance and environmental testing period at Raytheon, and showed no significant change. The primary purpose of the URA is to determine relative gain coefficients of detectors measuring orthogonal polarizations. Changes in these coefficients between measurements at Raytheon and with the APS installed in the Glory spacecraft were less than $0.1 \%$. Subsequent measurements of the relative gain coefficients using the URA during TVAC testing of the Glory spacecraft (Figure 2) continued to be show stable behavior with typical variations of less than $0.1 \%$, in those spectral bands with adequate signal, over the entire spacecraft level environmental testing period and $45^{\circ} \mathrm{C}$ temperature range $\left(-9 /+36^{\circ} \mathrm{C}\right)$ that the optics detector module $(\mathrm{ODM})$ was exposed to.
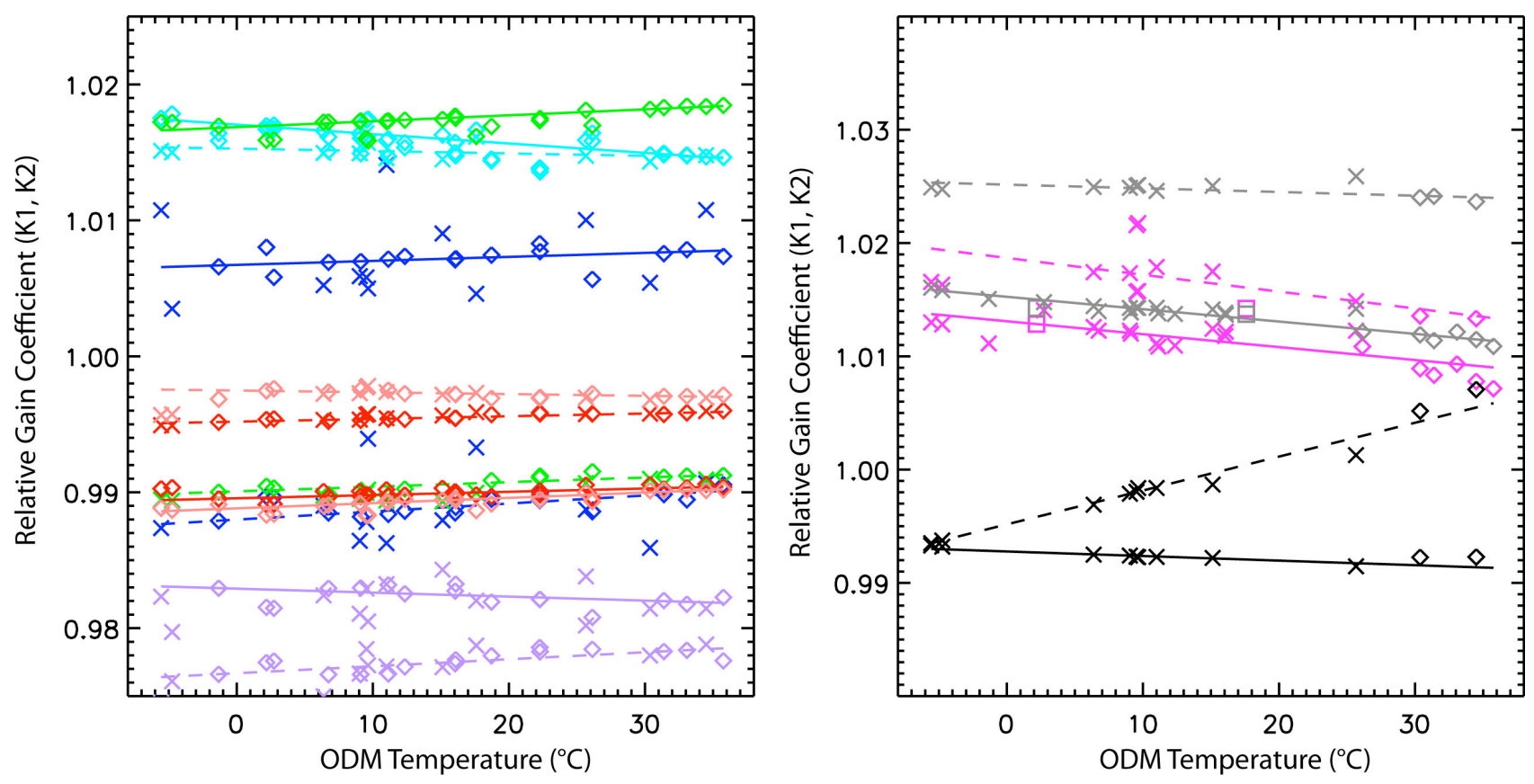

Figure 2: Example of the relative gain coefficient variation with ODM temperature during spacecraft level environmental testing for the VNIR bands $(410,443,555,670,865$ and $910 \mathrm{~nm}$ shown as blue, mauve, turquoise, green, red and orange respectively) in the left panel and the SWIR bands (1378, 1600 and $2260 \mathrm{~nm}$ shown as fuschia, grey and black respectively) in the right panel. Linear fits of the relative gain coefficients to ODM temperature are shown with solid lines for K1 coefficients and with dashed lines for K2 coefficients. In the left hand panel the diamonds indicate tests with a bright lamp level and crosses have a low lamp level. In the right panel the SWIR detectors are at a set point of $155 \mathrm{~K}$ (crosses), or $165 \mathrm{~K}$ (diamonds).

The lamps used during TVAC testing cannot provide a very bright signal for the blue bands $(410,443 \mathrm{~nm})$ which is why there is noise apparent in the determination of these relative gain coefficients, particularly in the low lamp level tests 
(crosses in left panel). The only relative gain coefficient that has a clear temperature dependence is the $2260 \mathrm{~nm} \mathrm{~K} 2$ coefficient which has a $0.03 \% /{ }^{\circ} \mathrm{C}$ variation over the $45^{\circ} \mathrm{C}$ range that was tested.

\subsection{Polarized reference assembly}

A second nadir-facing polarimetric calibrator, the Polarized Reference Assembly (PRA), contains a set of four GlanTaylor prisms for the VNIR bands and a single wire grid polarizer for the SWIR bands to create a view for all channels that is highly polarized with a known azimuth. The PRA is used to ensure that the absolute degree of linear polarization of scene measurements is correct. It is also used to monitor and correct for any change in instrumental polarization that may occur over the mission lifetime.

The APS internal PRA was tested in a similar procedure to that used for measuring the URA. The external polarized and unpolarized sources were used to determine the exact orientation of the Glan-Taylor and wire-grid polarizers in the PRA. This allows the polarimetric scale factors $\alpha_{\mathrm{q}}$ and $\alpha_{\mathrm{u}}$ that are used to o be obtained from observations of the PRA. The Glan-Taylor polarizers used to calibrate telescope pairs in the VNIR are not perfectly aligned with $90^{\circ}$ between their polarization axes (mis-alignments are $1^{\circ}$ for A-telescopes and $1.5^{\circ}$ for B-telescopes) and the DoLP observed looking at the two different polarizers is not therefore expected to be $100 \%$, and can indeed be greater than $100 \%$. Since the wire grid polarizer used in the PRA cover both SWIR telescopes, the DoLP observed by the SWIR telescopes should be close to $100 \%$, and is, to within $0.03 \%$. The primary measure of the PRA performance is the comparison of the polarimetric scale factors $\alpha_{q}$ and $\alpha_{u}$ determined from a complete polarimetric calibration and those obtained from the PRA and these agreed with one another over TVAC thermal environments to within $0.25 \%$.
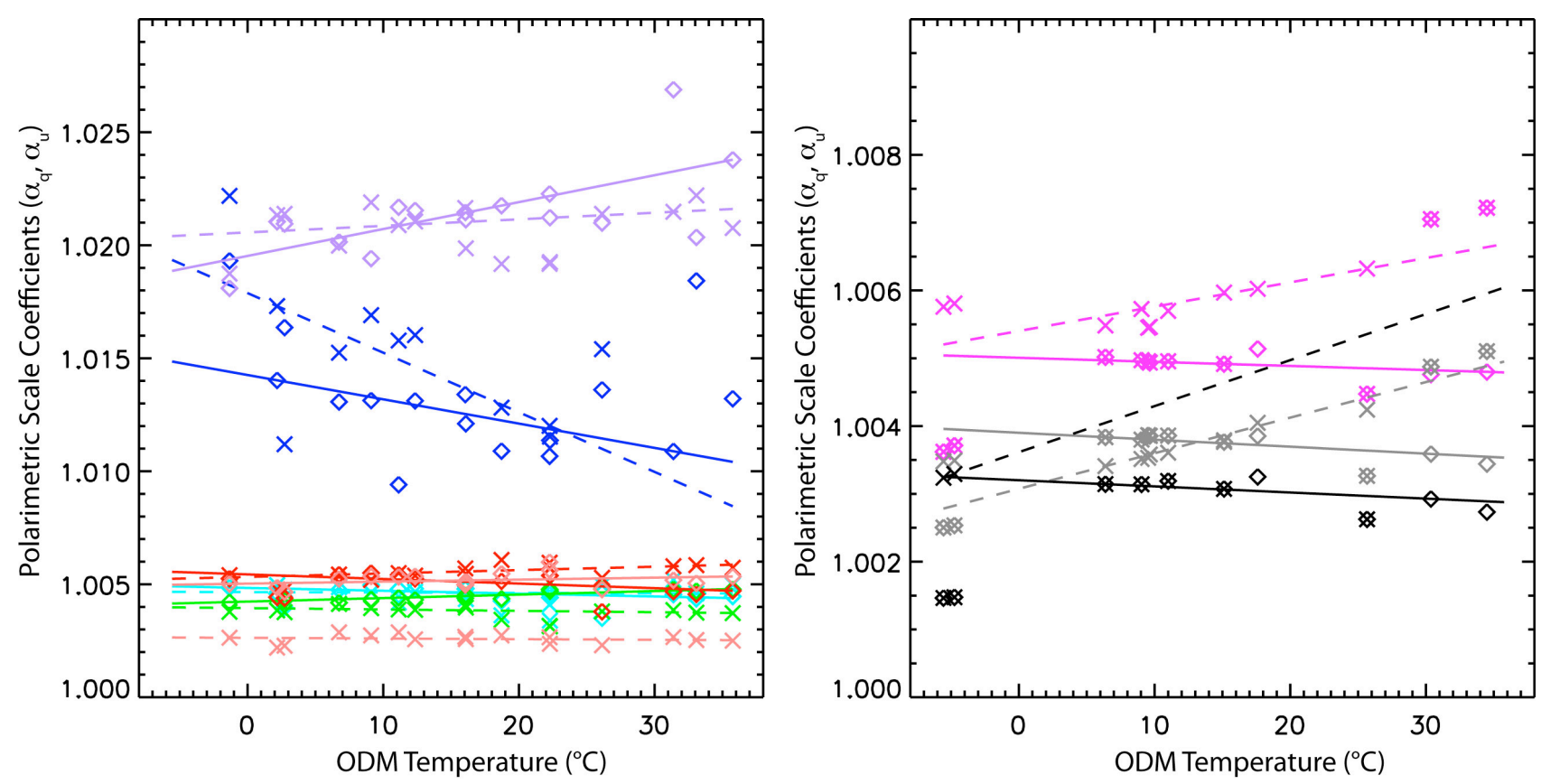

Figure 3: Variation in polarimetric scale coefficient determined with the PRA during environmental testing at the spacecraft level. Solid lines are for aq and dashed lines are for au. Colors and symbols represent different bands, lamp levels and detector temperatures as described in the caption for Fig 2.

The polarimetric scale factors continued to be stable to within $0.2 \%$ during TVAC testing of APS on the spacecraft, at least in those bands with an adequate signal (Figure 3). The bands with low signal levels (410 and $443 \mathrm{~nm}$ ) show no apparent variation in the polarimetric scale factor with ODM temperature and the noise in the determination of the coefficient is consistent with the available signal. Signal levels will not be an issue on orbit since the sun provides a much brighter source in the blue than a tungsten lamp. 


\subsection{Dark reference assembly}

The Dark Reference Assembly (DRA) is designed to extinguish stray light that is incident upon it. Tests were performed at Raytheon to characterize possible leakage of radiance into the DRA when the APS was viewing a bright scene (1000 W lamp) illuminating a Spectralon ${ }^{\mathrm{TM}}$ plaque. The maximum change in the APS Dark Reference signal did not exceed the maximum allowable $0.05 \%$ of scene radiance (APS requirement), when compared to a dark reference signal with zero scene radiance.

In addition the data taken during spacecraft TVAC testing, which included extremely bright illumination of the APS in the near field, was evaluated to determine whether any light was leaking into the dark reference. It was found there was less than $0.05 \mathrm{DN}$ effect on the dark reference, which is the limit of the accuracy of this characterization. This analysis used more than 100,000 scans (48 hours) of data, providing better signal to noise ratio than was available in the Raytheon test, and the worst case corresponds to a change in dark reference of less than $0.005 \%$ with an uncertainty of $\pm 0.005 \%$.

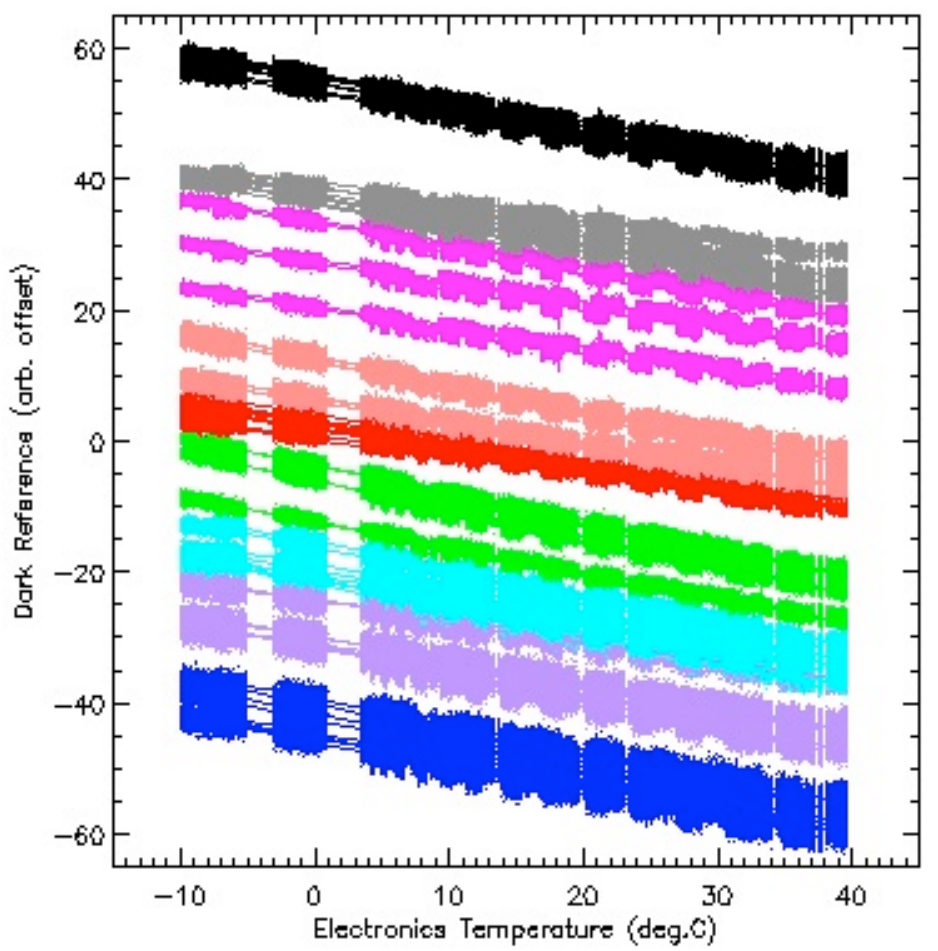

Figure 4: Changes in DRA signal as a function of electronics module temperature. Different spectral bands have arbitrary offsets to allow all bands to be displayed on the same figure.

The behavior of the dark reference level as a function of temperature was also evaluated during testing at Raytheon and also during spacecraft level testing and is shown in Figure 5. It was found that the dark reference level for a particular electronics temperature was stable to within $0.5 \mathrm{DN}$ over multiple TVAC temperature cycles and that the dependence on electronics temperature of the dark reference level was about $-0.3 \mathrm{DN} /{ }^{\circ} \mathrm{C}$ with some small variation between channels and bands. The temperature dependence for a particular channel estimated using different TVAC temperature cycles was stable to better than $0.01 \mathrm{DN} /{ }^{\circ} \mathrm{C}$. This information is of particular interest for dark reference subtraction since using dark reference data from any given scan only allows the dark reference level to be determined to within a few DN. Using a temperature dependent relationship to estimate the dark reference over a full orbit will allow the dark reference level to be determined to within a single DN. 


\subsection{Solar reference assembly}

The Solar Reference Assembly (SRA) provides for absolute radiometric correction by means of solar reflectance from a well-characterized Spectralon ${ }^{5}$ diffuser plate. The bi-directional reflectance distribution function (BRDF) and surface flatness was characterized on the ground so that the angularly dependent reflectance of the diffuser will not be confused with the sun's angle of incidence variations, on orbit.

\section{TIM DESIGN}

The TIM instrument uses four ambient temperature electrical substitution radiometers to measure the Sun's radiative input power over the entire solar spectrum. Each is located behind a precision NIST-calibrated aperture, which defines the area over which radiation is collected to provide the ratio of power to area, or irradiance. Instrument stability is maintained by duty cycling the four such that potential solar exposure dependent degradation in the primary radiometer, which views the Sun the majority of the time, can be tracked and corrected via inter-comparisons with the lesser-used ones, which remain closer to their ground calibration values. The SORCE TIM has shown less degradation than any other TSI instrument on-orbit and the Glory TIM benefits from the same robust radiometer design (described in [6]), so should achieve it's $0.001 \%$ /year stability requirement.

Calibration differences have caused offsets in absolute value between prior TSI instruments that have only been mitigated by overlap between successive missions. The Glory TIM is intended to have superior accuracy to any previously flown TSI instrument due to improved electronics, component-level ground calibrations, and new end-to-end irradiance validations. The newly created TSI Radiometer Facility (TRF), funded under the Glory program, provides the heretofore unavailable ability to perform end-to-end validations by directly comparing a TSI instrument to a reference NIST-calibrated cryogenic radiometer under flight-like operating conditions of measuring irradiance in vacuum and at full solar power levels. Recent validations of the Glory TIM on this facility indicate the TIM's goal of $0.01 \%$ absolute accuracy has been achieved, making the TSI climate data record more robust in the undesirable event of a gap in future measurements.

\section{SUMMARY}

Glory is an Earth-orbiting observatory designed to continue and improve upon long-term monitoring of two key forcings influencing global climate. One of its primary objectives is to determine the global distribution of aerosol and cloud properties with accuracy and coverage sufficient for reliable quantifications of the aerosol direct and indirect effects on climate. The other is to continue the 32-year TSI measurement record to help quantify the effects of solar variability on the Earth's climate. These objectives are met by implementing two separate science instruments. The APS has the ability to collect multi-angle photopolarimetric measurements of the atmosphere and the underlying surface along the satellite ground track within the visible, near-infrared, and short-wave infrared spectral region ranging from 400 to $2200 \mathrm{~nm}$. Based on a proven technique demonstrated by aircraft instruments, Glory APS observations will provide extremely accurate retrievals of aerosol climate forcing parameters and are expected to improve global aerosol assessments with other A-Train instruments. They will also pave the way for future operational results by providing proof of concept and risk reduction and demonstrating synergy with imagery offered by radiometers like the VIIRS instrument to be flown on the NPOESS Preparatory Project (NPP).

The TIM will measure the incident sunlight at the top of the Earth's atmosphere by collecting high accuracy and precision TSI data. The Glory TIM is an improved version of the proven TIM currently flying on SORCE, and overlap with this mission will maintain continuity of the 32-year benchmark TSI record used in determining climate sensitivities to solar variability.

The Glory Science Team was recently selected on the basis of competitive proposals and their efforts are aimed at improving, validating, and assimilating the Glory data products and using them in climate studies. The scientific knowledge provided by the Glory mission will be essential to understanding climate change for sound, scientifically- 
based economic and policy decisions related to environmental changes caused by climate variability as well as offering significant improvements in operational weather observations and forecasting. Subsequent launches of both Glory instruments would serve to continue their benefits to operational weather as well as climate trend assessments well beyond the initial Glory mission demonstration.

\section{ACKNOWLEDGEMENTS}

The Glory project was funded by, and is part of, the NASA EOS project. We appreciate all the help from the entire Glory project, including the understanding of the families of the many staff members who have worked tirelessly through weekends and vacations to allow the project to come to fruition.

\section{REFERENCES}

[1] G. Kopp, G. Lawrence, and G. Rottman, "The Total Irradiance Monitor (TIM): Science Results," Solar Physics, 230, 1, Aug. 2005, pp. 129-140.

[2] M. I. Mishchenko et al., "Accurate monitoring of terrestrial aerosols and total solar irradiance: introducing the Glory mission." Bull. Amer. Meteorol. Soc., 88, pp. 677-691, 2007.

[3] Peralta, R., et al., "Aerosol Polarimetry Sensor for the Glory Mission”, SPIE Proceedings, 6786, 2007.

[4] Persh, S. et al., "Ground Performance Measurements of the Glory Aerosol Polarimetry Sensor", SPIE Proceedings, 7807-2, 2010.

[5] G. T. Georgiev, et al., "BRDF Study of Gray-Scale SPECTRALON”, SPIE Proceedings, 7081, 2008.

[6] G. Kopp and G. Lawrence, "The Total Irradiance Monitor (TIM): Instrument Design," Solar Physics, 230, 1, Aug. 2005, pp. 91-109. 